\title{
Modified Crack Detection of Sewer Conduit with Low Resolution Images
}

\author{
Son, Byung Jik ${ }^{1} \cdot$ Cho, Tae Jun ${ }^{2}$ \\ ${ }^{2}$ Professor, Department of Civil engineering, Daejin University, Pocheon, Korea
}

\begin{abstract}
Imaging devices of less than 300,000 pixels are mostly used for sewage conduit exploration due to the petty nature of the survey industry in Korea. Particularly, devices of less than 100,000 pixels are still widely used, and the environment for image processing is very bitter. Since the sewage conduit images covered in this study have a very low resolution $(240 \times 320=76,800$ pixels $)$, it is very difficult to detect cracks. Because most of the resolution of the sewer conduit images are very low in Korea, this problem of low resolution was selected as the subject of study. Cracks were detected through a total of six steps of improving the crack in Step 2, finding the optimal threshold value in Step 3, and applying an algorithm to detect cracks in Step 5. Cracks were effectively detected by the optimal parameters in Steps 2 and 3 and the user algorithm in Step 5. Despite the very low resolution, the cracked images showed $96.4 \%$ accuracy of detection, and the non-cracked images showed $94.5 \%$ accuracy. Moreover, the analysis was excellent in quality, also. It is believed that the findings of this study can be effectively used for crack detection with low-resolution images.
\end{abstract}

Key Words: image processing, low resolution image, crack detection, user algorithm

\section{Introduction}

The recent urban sinkholes in Korea are often caused by the loss of surrounding soil due to the aging of sewer built intensively in the $70 \mathrm{~s}$ and $80 \mathrm{~s}$. In the case of Korea, 980 local governments nationwide are conducting a detailed investigation on $12,000 \mathrm{~km}$ of the entire sewer, which is over 20 years old, to prevent sinkholes.

Since the current structure maintenance technique evaluates the condition with the naked eye of the inspector based on manpower, a lot of time and effort is consumed, resulting in poor objectivity and reliability of the survey. One of the ways to compensate for this problem is to use digital image processing technology.

Recently, studies on the introduction of an image processing technique capable of analyzing CCTV images of sewer or 
the development of the devices are being conducted. Xu et al. (1998) measured the deformation of the sewer through image analysis using a probe equipped with an annular laser pointer. McKim and Sinha (1999) extracted information on cracks in sewer using basic image processing techniques, and Moselhi and Shehab-Eldeen (1999) applied artificial neural network method in addition. Later, Yang and Su (2008) studied an advanced image processing method applying machine learning, and Yang et al. (2011) also proposed a method for evaluating the quality of images taken from the sewer. However, there is a limit to the application of the developed image processing method because the quality of the image taken while moving in such a dark environment with a lot of obstacles as the sewer is very low.

As for the sewer exploration device, a product using a high-resolution digital camera of more than 2 million pixels has been developed. However, devices of less than 300,000 pixels are mostly being used in Korea because of the small size of the Korean survey industry and still low awareness of the severity of the sewer problem in Korea.

The sewer conduit images covered in this study are of 76,800 pixels $(240 \times 320)$, and it is difficult to detect cracks at such low resolution. Son et al. $(2017,2019)$ studied the crack detection method of these low-resolution sewer images twice in 2017 and 2019. Their 2019 study showed relatively high accuracy research results, but it is a bit complicated to apply. An investigation of improving crack detection to compensate for these problems was conducted in this study.

\section{Crack detection procedure}

As shown in Table 1, the sewer CCTV image is a video clip of 200 second duration in very low-resolution images of 76,800 pixels $(240 \times 320)$. A total of 110 images were selected as analysis targets, excluding unnecessary (such as the beginning of the video clip) and overlapping images from a total of 200 images, one image per second. Of the $110 \mathrm{images,}$ both cracked images (c1 c55) and non-cracked images (n1 n55) counted to 55 each, and 20 sample data are shown in Figures 1 and 2 .

The crack detection procedure for low-resolution images goes through six steps as shown in Table 2 and Fig. 3. The important steps in this procedure are Steps 2, 3, and 5, and the crack detection process for each step is summarized as follows.

\subsection{Step 1: Read the Image}

This is the stage of reading the original video of the sewer. Table 2 shows c18 and n 25 as examples among 55 cracked images and 55 non-cracked images.

\subsection{Step 2: Enhance the Image}

First, a color image is converted into a grayscale image, and the image is enhanced in this step. There are many ways to enhance the image. This study applied the method of Contrast-Limited Adaptive Histogram Equalization (CLAHE) proposed by Zuiderveld (1994). CLAHE is a method widely used for contrast enhancement. Among the various parameters of CLAHE, an important parameter in this study is ClipLimit. The smaller the ClipLimit is, the more the histogram value redistributed from the maximum value increases, resulting in a more pronounced contrast. The range is a real number between 0 and 1 , and the example in Table 2 uses 0 . The parameter study of changing ClipLimit between 0 and 1 is discussed in Chapter 3. 
Table 1. Specification of sewer video clip

\begin{tabular}{c|c}
\hline Duration & $200 \mathrm{sec}$ \\
\hline Frame Rate & 30 \\
\hline Height & 240 \\
\hline Width & 320 \\
\hline
\end{tabular}
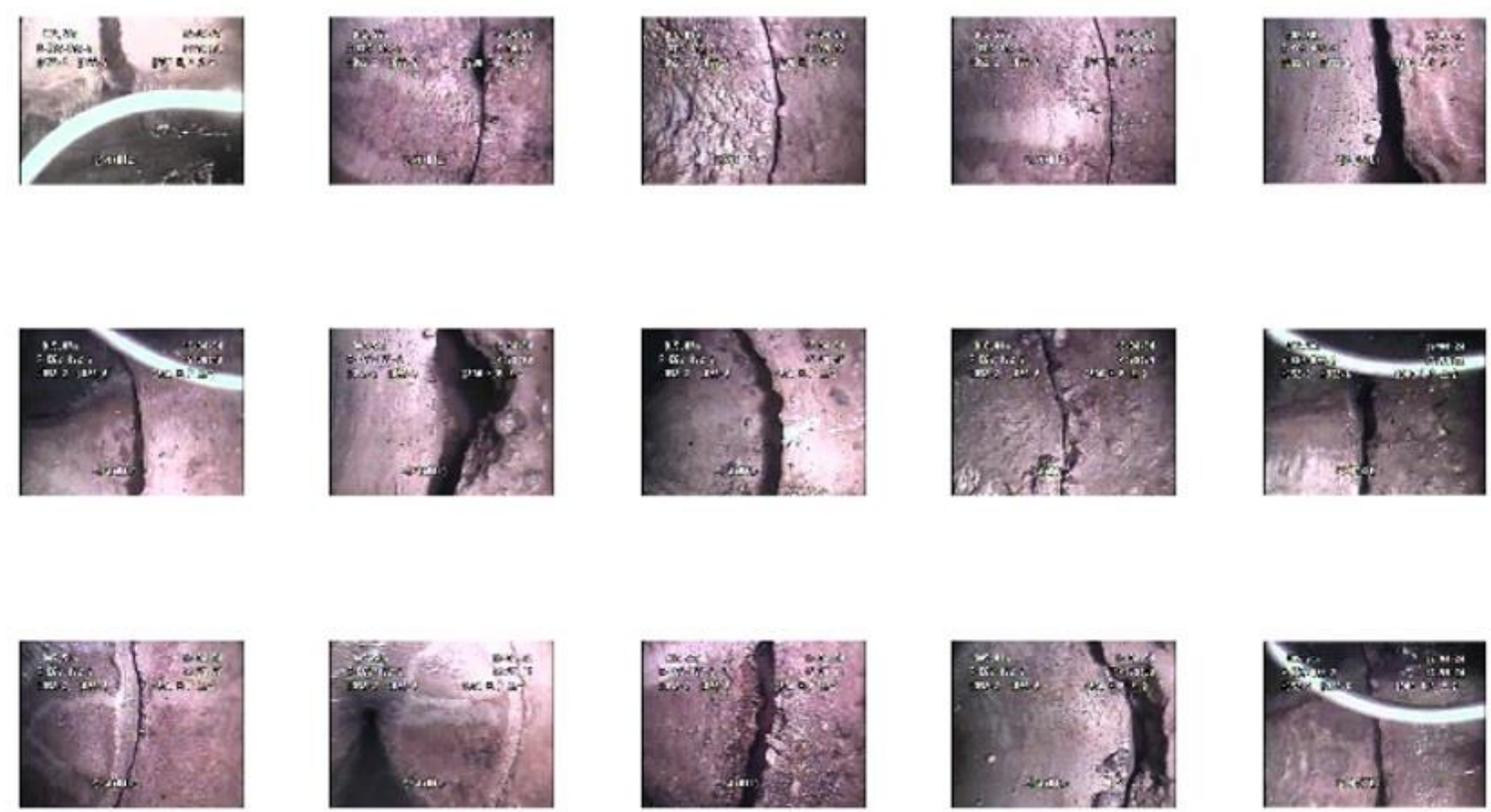

Fig. 1 Sample images of cracks 

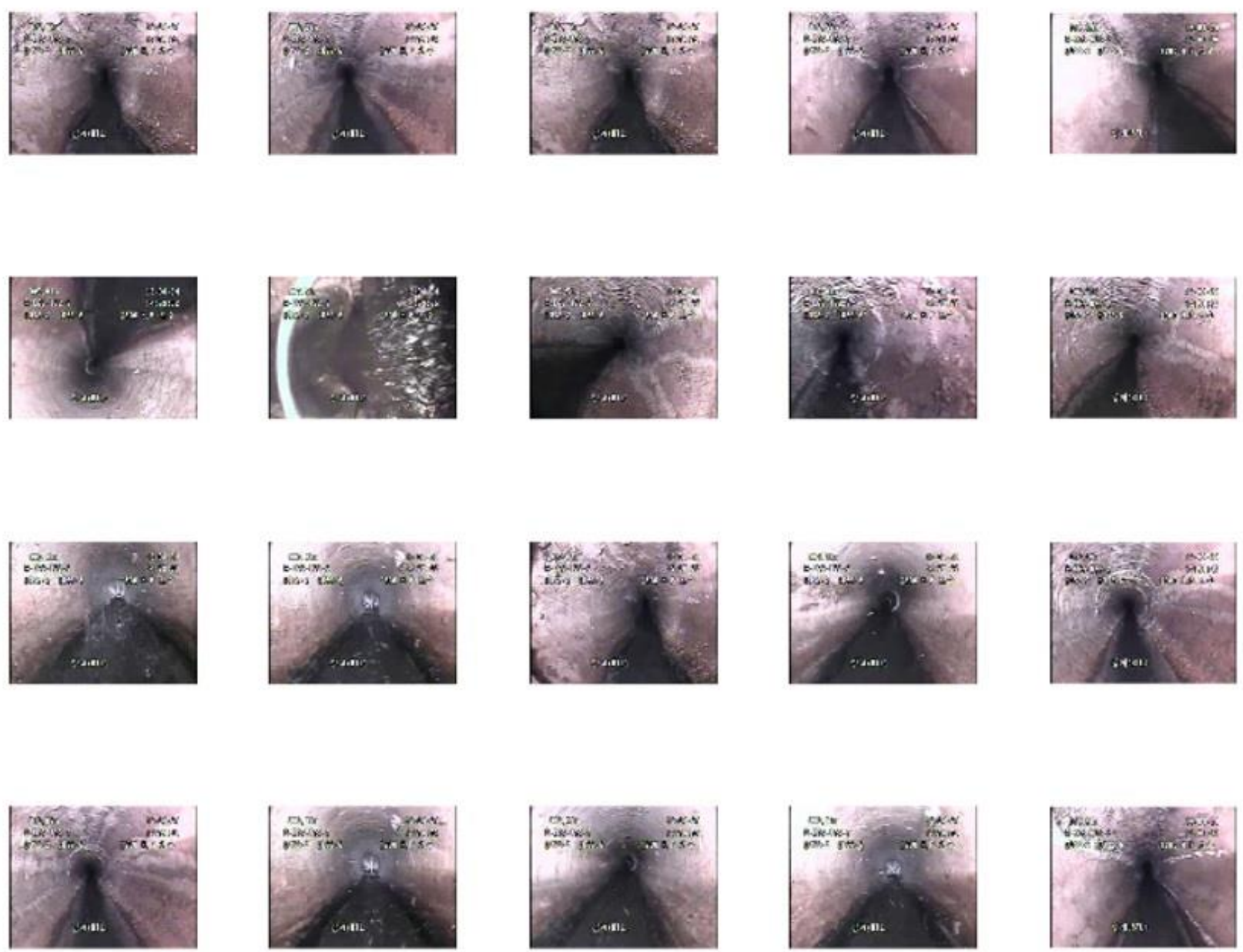

Fig. 2 Sample images of non-cracks

\subsection{Step 3: Make the Binary Image}

This step converts analog images to binary images. For crack detection, it is necessary to find an optimal threshold value. The method proposed by Bradley (2007) was used to compute it, and the parameters include sensitivity and statistical analysis method.

Table 2 Crack detection procedure

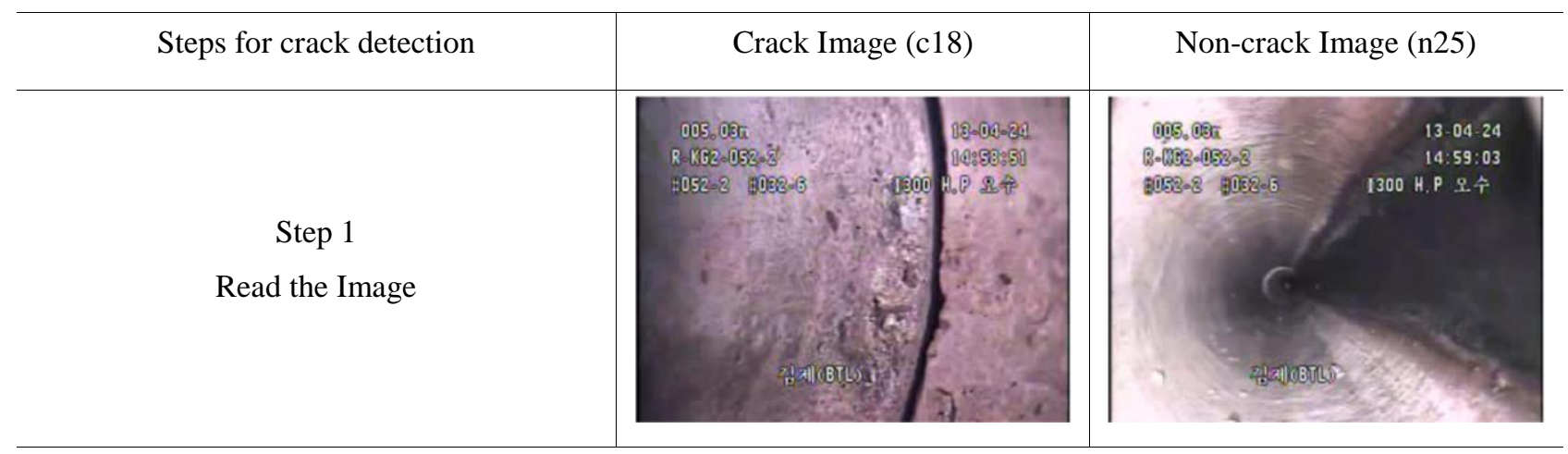




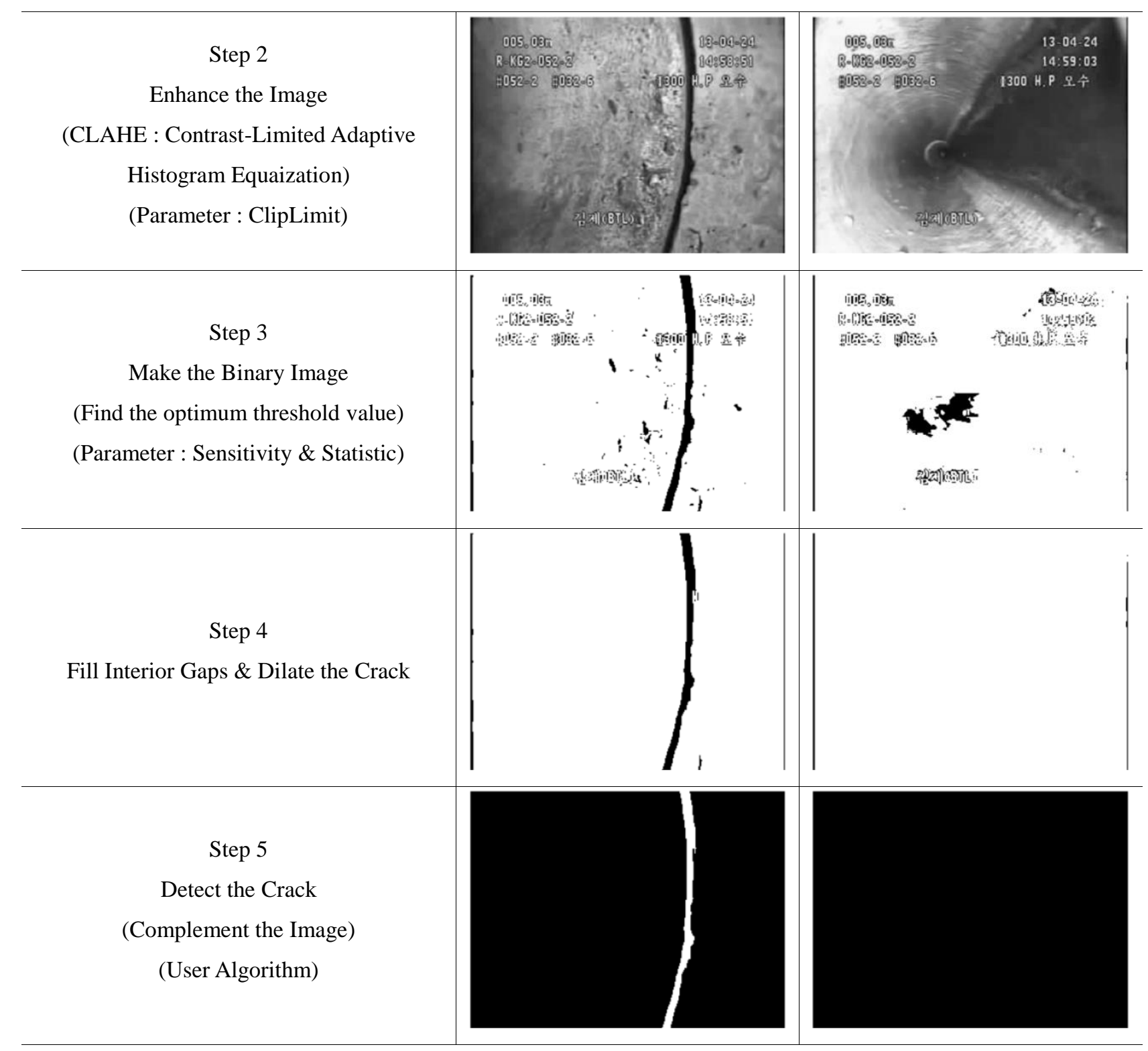




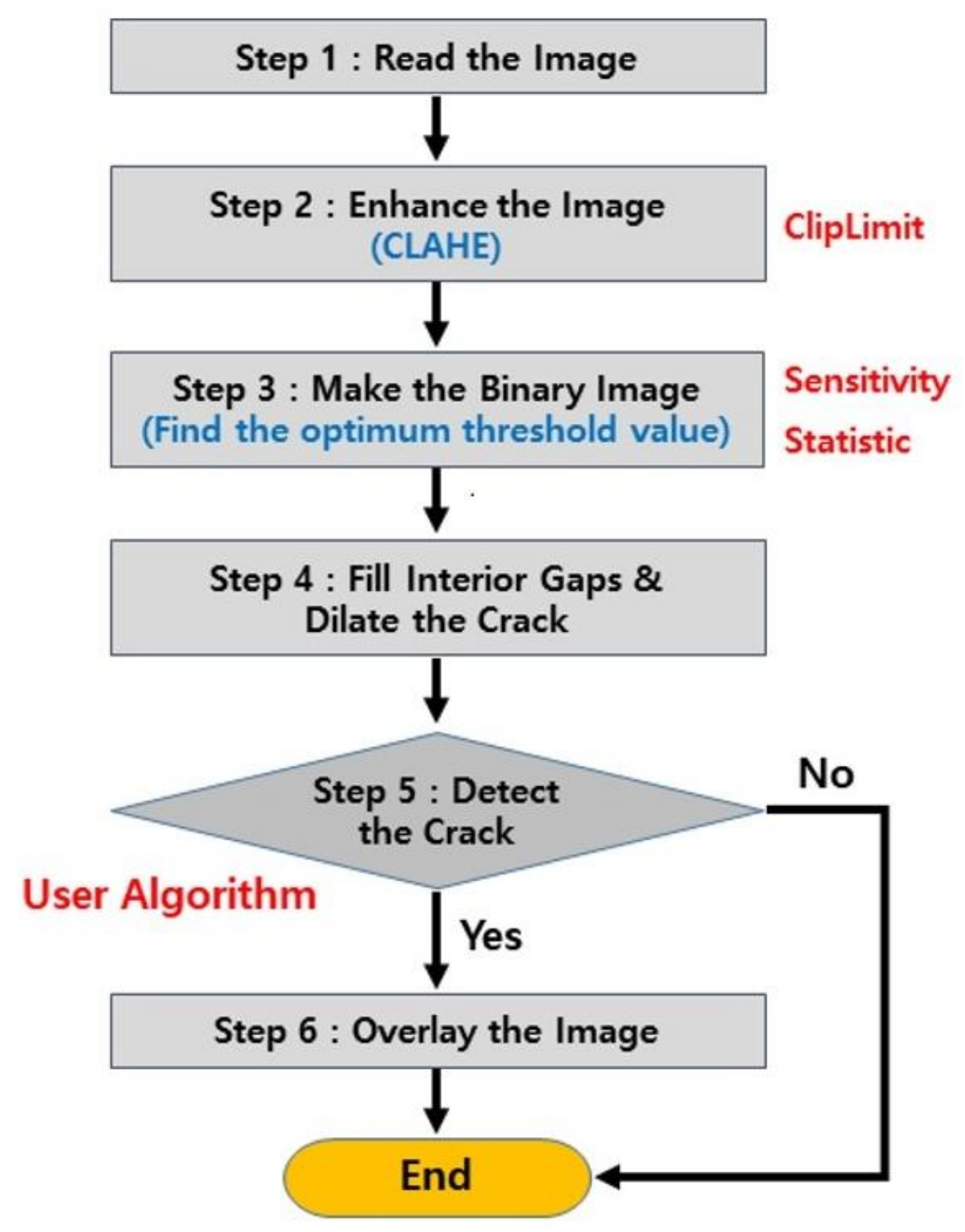

Fig. 3 Flowchart of crack detection

Sensitivity assigns a real number between 0 and 1 . If the value is large, there is a risk of including some background pixels. Despite the risk, the larger pixels are regarded as a foreground for the binary conversion. Statistical methods can be selected from mean, median, or Gaussian. Mean is the local average value of the neighborhood, median is the local median of the neighborhood, and Gaussian uses the Gaussian weighted average of the neighborhood as a statistic.

In the example of Table 2, the sensitivity was 1 , and the chosen statistic was Gaussian. The parameter study of sensitivity and statistical method is conducted in Chapter 3.

2.4 Step 4: Fill Interior Gaps and Dilate the Crack

This step involves filling the inside of the crack and expanding the crack. The method proposed by Soille (1999) was used to 
fill the inside of the crack, and cracks remain mostly. Any deformation, that is not a crack, can be removed through the following step. The image dilation method proposed by Gonzalez et al. (2009) was applied to highlight the cracks.

\subsection{Step 5: Detect the Crack}

This step determines the presence or absence of cracks, i.e., extracting only the cracks. It determines whether the object filled in the interior in Step 4 is a crack. In this step, a User Algorithm is required and will be explained in detail in Chapter 3. Additionally, it is a step of inverting the black and white of the image to improve readability.

\subsection{Step 6: Overlay the Image}

Finally, it is the step of overlaying the detected crack boundary on the original image. Gonzalez et al. (2009) proposed method was used.

\section{Crack detection parameters and user algor ithm}

Finally, this step overlays the detected crack boundary on the original image by the method of Gonzalez et al. (2009).

\subsection{Crack detection parameters}

Table 3 shows the analysis parameters described in Chapter 2, and Tables 4 to 6 show the analysis results. (a) The result of a crack image refers to the accuracy of finding cracks, and (b) the result of a non-crack image refers to the accuracy of finding non-crack. The smaller the sensitivity, the accuracy of the non-cracked image gets close to $100 \%$, but the accuracy of the cracked image gets closer to $0 \%$. The greater the sensitivity (closer to 1), the higher the accuracy of both cracked and non-cracked images. The reason for this is that, although there is a risk of including some background pixels as the sensitivity increases, more pixels are regarded as a foreground to be binarized. Additionally, it is analyzed that the smaller the value of ClipLimit is, the more pronounced the contrast becomes, and thus showing better results.

Table 3 Analysis parameters

\begin{tabular}{c|c|c}
\hline Var. \# & Name & Range \\
\hline Var.\#1 & ClipLimit & $\begin{array}{c}0,0.00001,0.0001,0.01, \\
{[0.1: 0.1: 1](15)}\end{array}$ \\
\hline Var. \#2 & Sensitivity & $\begin{array}{c}{[0: 0.1: 1] \text { (Start: Spacing: End) }} \\
(11)\end{array}$ \\
\hline Var.\#3 & Statistic & Mean, Median, Gaussian \\
\hline
\end{tabular}


Table 4 Analysis results (\%) by Var. \#1 vs. Var. \#2 (Var. \#3: Mean)

\begin{tabular}{|c|c|c|c|c|c|c|c|c|c|c|c|c|}
\hline & Var. \#1 & 0.0 & 0.1 & 0.2 & 0.3 & 0.4 & 0.5 & 0.6 & 0.7 & 0.8 & 0.9 & 1.0 \\
\hline & 0 & 0.0 & 0.0 & 0.0 & 0.0 & 0.0 & 3.6 & $\begin{array}{l}87 . \\
3\end{array}$ & $\begin{array}{l}92 . \\
7\end{array}$ & $\begin{array}{l}96 . \\
4\end{array}$ & $\begin{array}{l}94 . \\
5\end{array}$ & $\begin{array}{l}94 . \\
5\end{array}$ \\
\hline & 0.00001 & 0.0 & 0.0 & 0.0 & 0.0 & 0.0 & 3.6 & $\begin{array}{l}87 . \\
3\end{array}$ & $\begin{array}{l}92 . \\
7\end{array}$ & $\begin{array}{l}96 . \\
4\end{array}$ & $\begin{array}{l}94 . \\
5\end{array}$ & $\begin{array}{l}94 . \\
5\end{array}$ \\
\hline & 0.0001 & 0.0 & 0.0 & 0.0 & 0.0 & 0.0 & 3.6 & $\begin{array}{l}87 . \\
3\end{array}$ & $\begin{array}{l}92 . \\
7\end{array}$ & $\begin{array}{l}96 . \\
4\end{array}$ & $\begin{array}{l}94 . \\
5\end{array}$ & $\begin{array}{l}94 . \\
5\end{array}$ \\
\hline & 0.001 & 0.0 & 0.0 & 0.0 & 0.0 & 1.8 & 5.5 & $\begin{array}{l}78 . \\
2\end{array}$ & $\begin{array}{l}90 . \\
9\end{array}$ & $\begin{array}{l}96 . \\
4\end{array}$ & $\begin{array}{l}94 . \\
5\end{array}$ & $\begin{array}{l}94 . \\
5\end{array}$ \\
\hline & 0.01 & 0.0 & 0.0 & 0.0 & 0.0 & 0.0 & 5.5 & $\begin{array}{l}52 . \\
7\end{array}$ & $\begin{array}{l}92 . \\
7\end{array}$ & $\begin{array}{l}98 . \\
2\end{array}$ & $\begin{array}{c}10 \\
0.0\end{array}$ & $\begin{array}{l}98 . \\
2\end{array}$ \\
\hline & 0.1 & 0.0 & 0.0 & 0.0 & 0.0 & 0.0 & 5.5 & $\begin{array}{l}45 . \\
5\end{array}$ & $\begin{array}{l}90 . \\
9\end{array}$ & $\begin{array}{l}98 . \\
2\end{array}$ & $\begin{array}{c}10 \\
0.0\end{array}$ & $\begin{array}{c}10 \\
0.0\end{array}$ \\
\hline (a) & 0.2 & 0.0 & 0.0 & 0.0 & 0.0 & 0.0 & 5.5 & $\begin{array}{l}47 . \\
3\end{array}$ & $\begin{array}{l}90 . \\
9\end{array}$ & $\begin{array}{l}98 . \\
2\end{array}$ & $\begin{array}{c}10 \\
0.0\end{array}$ & $\begin{array}{c}10 \\
0.0\end{array}$ \\
\hline $\begin{array}{l}\text { Crack } \\
\text { Images }\end{array}$ & 0.3 & 0.0 & 0.0 & 0.0 & 0.0 & 0.0 & 5.5 & $\begin{array}{l}49 . \\
1\end{array}$ & $\begin{array}{l}90 . \\
9\end{array}$ & $\begin{array}{l}98 . \\
2\end{array}$ & $\begin{array}{r}10 \\
0.0\end{array}$ & $\begin{array}{c}10 \\
0.0\end{array}$ \\
\hline & 0.4 & 0.0 & 0.0 & 0.0 & 0.0 & 0.0 & 5.5 & $\begin{array}{l}49 . \\
1\end{array}$ & $\begin{array}{l}90 . \\
9\end{array}$ & $\begin{array}{l}98 . \\
2\end{array}$ & $\begin{array}{r}10 \\
0.0\end{array}$ & $\begin{array}{c}10 \\
0.0\end{array}$ \\
\hline & 0.5 & 0.0 & 0.0 & 0.0 & 0.0 & 0.0 & 5.5 & $\begin{array}{l}49 . \\
1\end{array}$ & $\begin{array}{l}90 . \\
9\end{array}$ & $\begin{array}{l}98 . \\
2\end{array}$ & $\begin{array}{c}10 \\
0.0\end{array}$ & $\begin{array}{l}10 \\
0.0\end{array}$ \\
\hline & 0.6 & 0.0 & 0.0 & 0.0 & 0.0 & 0.0 & 5.5 & $\begin{array}{l}49 . \\
1\end{array}$ & $\begin{array}{l}90 . \\
9\end{array}$ & $\begin{array}{l}98 . \\
2\end{array}$ & $\begin{array}{c}10 \\
0.0\end{array}$ & $\begin{array}{l}10 \\
0.0\end{array}$ \\
\hline & 0.7 & 0.0 & 0.0 & 0.0 & 0.0 & 0.0 & 5.5 & $\begin{array}{l}49 . \\
1\end{array}$ & $\begin{array}{l}90 . \\
9\end{array}$ & $\begin{array}{l}98 . \\
2\end{array}$ & $\begin{array}{c}10 \\
0.0\end{array}$ & $\begin{array}{c}10 \\
0.0\end{array}$ \\
\hline & 0.8 & 0.0 & 0.0 & 0.0 & 0.0 & 0.0 & 5.5 & $\begin{array}{l}49 . \\
1\end{array}$ & $\begin{array}{l}90 . \\
9\end{array}$ & $\begin{array}{l}98 . \\
2\end{array}$ & $\begin{array}{r}10 \\
0.0\end{array}$ & $\begin{array}{c}10 \\
0.0\end{array}$ \\
\hline & 0.9 & 0.0 & 0.0 & 0.0 & 0.0 & 0.0 & 5.5 & $\begin{array}{l}49 . \\
1\end{array}$ & $\begin{array}{l}90 . \\
9\end{array}$ & $\begin{array}{l}98 . \\
2\end{array}$ & $\begin{array}{c}10 \\
0.0\end{array}$ & $\begin{array}{c}10 \\
0.0\end{array}$ \\
\hline & 1.0 & 0.0 & 0.0 & 0.0 & 0.0 & 0.0 & 5.5 & $\begin{array}{l}49 . \\
1\end{array}$ & $\begin{array}{l}90 . \\
9\end{array}$ & $\begin{array}{l}98 . \\
2\end{array}$ & $\begin{array}{c}10 \\
0.0\end{array}$ & $\begin{array}{c}10 \\
0.0\end{array}$ \\
\hline
\end{tabular}




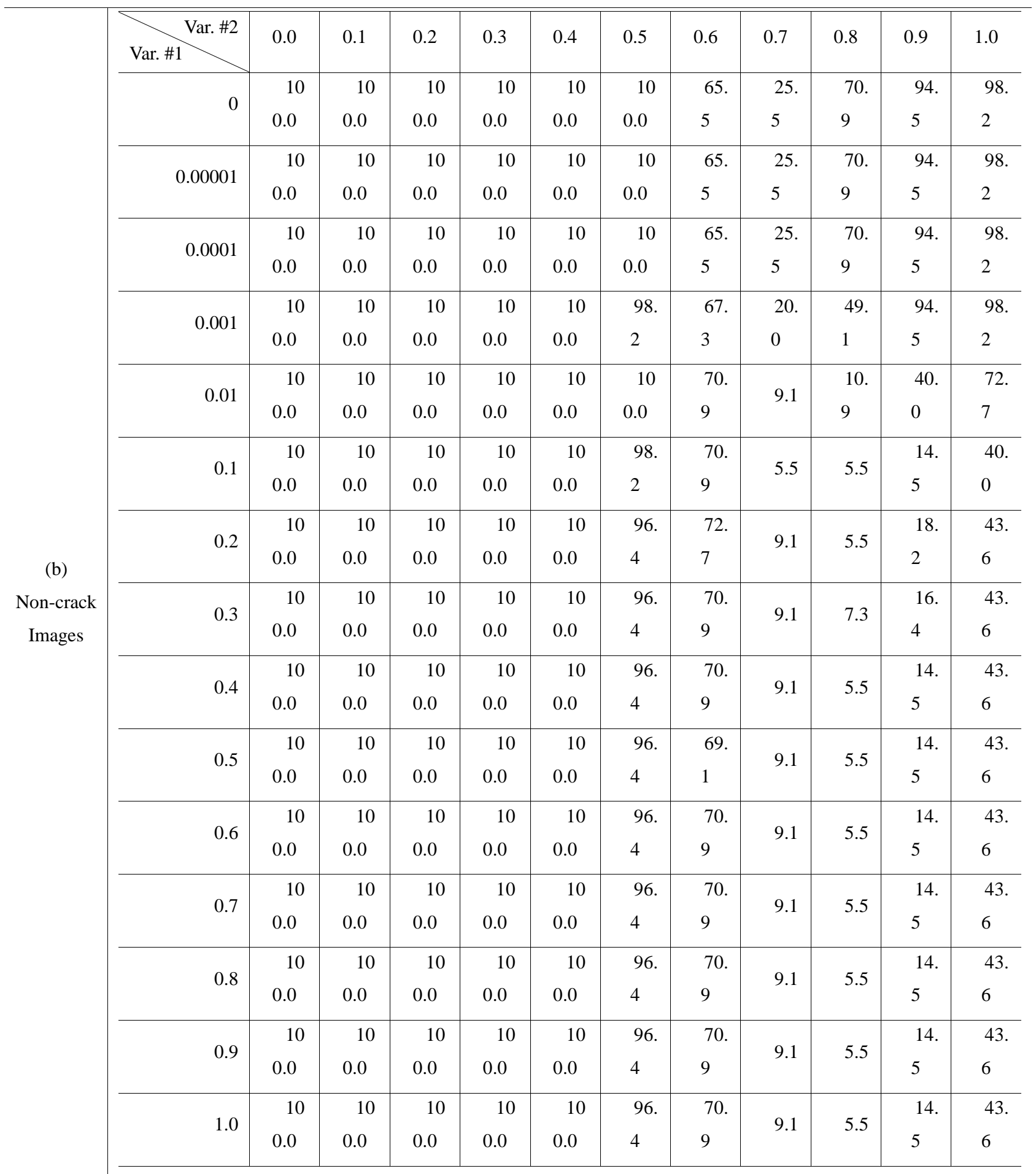


Table 5 Analysis results (\%) by Var. \#1 vs. Var. \#2 (Var. \#3: Median)

\begin{tabular}{|c|c|c|c|c|c|c|c|c|c|c|c|c|}
\hline & Var. \#1 & 0.0 & 0.1 & 0.2 & 0.3 & 0.4 & 0.5 & 0.6 & 0.7 & 0.8 & 0.9 & 1.0 \\
\hline & 0 & 0.0 & 0.0 & 0.0 & 0.0 & 0.0 & 0.0 & $\begin{array}{l}49 . \\
1\end{array}$ & $\begin{array}{l}98 . \\
2\end{array}$ & $\begin{array}{l}94 . \\
5\end{array}$ & $\begin{array}{l}87 . \\
3\end{array}$ & $\begin{array}{l}83 . \\
6\end{array}$ \\
\hline & 0.00001 & 0.0 & 0.0 & 0.0 & 0.0 & 0.0 & 0.0 & $\begin{array}{l}49 . \\
1\end{array}$ & $\begin{array}{l}98 . \\
2\end{array}$ & $\begin{array}{l}94 . \\
5\end{array}$ & $\begin{array}{l}87 . \\
3\end{array}$ & $\begin{array}{l}83 . \\
6\end{array}$ \\
\hline & 0.0001 & 0.0 & 0.0 & 0.0 & 0.0 & 0.0 & 0.0 & $\begin{array}{l}49 . \\
1\end{array}$ & $\begin{array}{l}98 . \\
2\end{array}$ & $\begin{array}{l}94 . \\
5\end{array}$ & $\begin{array}{l}87 . \\
3\end{array}$ & $\begin{array}{l}83 . \\
6\end{array}$ \\
\hline & 0.001 & 0.0 & 0.0 & 0.0 & 0.0 & 0.0 & 0.0 & $\begin{array}{l}41 . \\
8\end{array}$ & $\begin{array}{l}98 . \\
2\end{array}$ & $\begin{array}{l}94 . \\
5\end{array}$ & $\begin{array}{l}92 . \\
7\end{array}$ & $\begin{array}{l}85 . \\
5\end{array}$ \\
\hline & 0.01 & 0.0 & 0.0 & 0.0 & 0.0 & 0.0 & 0.0 & $\begin{array}{l}34 . \\
5\end{array}$ & $\begin{array}{l}96 . \\
4\end{array}$ & $\begin{array}{l}98 . \\
2\end{array}$ & $\begin{array}{c}10 \\
0.0\end{array}$ & $\begin{array}{l}90 . \\
9\end{array}$ \\
\hline & 0.1 & 0.0 & 0.0 & 0.0 & 0.0 & 0.0 & 1.8 & $\begin{array}{l}29 . \\
1\end{array}$ & $\begin{array}{l}90 . \\
9\end{array}$ & $\begin{array}{l}98 . \\
2\end{array}$ & $\begin{array}{l}10 \\
0.0\end{array}$ & $\begin{array}{l}96 . \\
4\end{array}$ \\
\hline (a) & 0.2 & 0.0 & 0.0 & 0.0 & 0.0 & 0.0 & 1.8 & $\begin{array}{l}27 . \\
3\end{array}$ & $\begin{array}{l}92 . \\
7\end{array}$ & $\begin{array}{l}10 \\
0.0\end{array}$ & $\begin{array}{c}10 \\
0.0\end{array}$ & $\begin{array}{l}96 . \\
4\end{array}$ \\
\hline $\begin{array}{l}\text { Crack } \\
\text { Images }\end{array}$ & 0.3 & 0.0 & 0.0 & 0.0 & 0.0 & 0.0 & 1.8 & $\begin{array}{l}29 . \\
1\end{array}$ & $\begin{array}{l}92 . \\
7\end{array}$ & $\begin{array}{l}10 \\
0.0\end{array}$ & $\begin{array}{l}10 \\
0.0\end{array}$ & $\begin{array}{l}96 . \\
4\end{array}$ \\
\hline & 0.4 & 0.0 & 0.0 & 0.0 & 0.0 & 0.0 & 1.8 & $\begin{array}{l}29 . \\
1\end{array}$ & $\begin{array}{l}92 . \\
7\end{array}$ & $\begin{array}{l}10 \\
0.0\end{array}$ & $\begin{array}{l}10 \\
0.0\end{array}$ & $\begin{array}{l}96 . \\
4\end{array}$ \\
\hline & 0.5 & 0.0 & 0.0 & 0.0 & 0.0 & 0.0 & 1.8 & $\begin{array}{l}29 . \\
1\end{array}$ & $\begin{array}{l}92 . \\
7\end{array}$ & $\begin{array}{c}10 \\
0.0\end{array}$ & $\begin{array}{c}10 \\
0.0\end{array}$ & $\begin{array}{l}96 . \\
4\end{array}$ \\
\hline & 0.6 & 0.0 & 0.0 & 0.0 & 0.0 & 0.0 & 1.8 & $\begin{array}{l}29 . \\
1\end{array}$ & $\begin{array}{l}92 . \\
7\end{array}$ & $\begin{array}{c}10 \\
0.0\end{array}$ & $\begin{array}{c}10 \\
0.0\end{array}$ & $\begin{array}{l}96 . \\
4\end{array}$ \\
\hline & 0.7 & 0.0 & 0.0 & 0.0 & 0.0 & 0.0 & 1.8 & $\begin{array}{l}29 . \\
1\end{array}$ & $\begin{array}{l}92 . \\
7\end{array}$ & $\begin{array}{c}10 \\
0.0\end{array}$ & $\begin{array}{c}10 \\
0.0\end{array}$ & $\begin{array}{l}96 . \\
4\end{array}$ \\
\hline & 0.8 & 0.0 & 0.0 & 0.0 & 0.0 & 0.0 & 1.8 & $\begin{array}{l}29 . \\
1\end{array}$ & $\begin{array}{l}92 . \\
7\end{array}$ & $\begin{array}{r}10 \\
0.0\end{array}$ & $\begin{array}{r}10 \\
0.0\end{array}$ & $\begin{array}{l}96 . \\
4\end{array}$ \\
\hline & 0.9 & 0.0 & 0.0 & 0.0 & 0.0 & 0.0 & 1.8 & $\begin{array}{l}29 . \\
1\end{array}$ & $\begin{array}{l}92 . \\
7\end{array}$ & $\begin{array}{r}10 \\
0.0\end{array}$ & $\begin{array}{r}10 \\
0.0\end{array}$ & $\begin{array}{l}96 . \\
4\end{array}$ \\
\hline & 1.0 & 0.0 & 0.0 & 0.0 & 0.0 & 0.0 & 1.8 & $\begin{array}{l}29 . \\
1\end{array}$ & $\begin{array}{l}92 . \\
7\end{array}$ & $\begin{array}{r}10 \\
0.0\end{array}$ & $\begin{array}{r}10 \\
0.0\end{array}$ & $\begin{array}{l}96 . \\
4\end{array}$ \\
\hline
\end{tabular}




\begin{tabular}{|c|c|c|c|c|c|c|c|c|c|c|c|c|}
\hline & Var. \#1 & 0.0 & 0.1 & 0.2 & 0.3 & 0.4 & 0.5 & 0.6 & 0.7 & 0.8 & 0.9 & 1.0 \\
\hline & 0 & $0.0^{10}$ & 0.0 & $0.0^{10}$ & $0.0^{10}$ & $0.0^{10}$ & $0.0^{10}$ & $\begin{array}{l}72 . \\
7\end{array}$ & $\begin{array}{l}32 . \\
7\end{array}$ & 90. & $0.0^{10}$ & $0.0^{10}$ \\
\hline & 0.00001 & $0.0^{10}$ & 0.0 & $0.0^{10}$ & $\begin{array}{l}10 \\
0.0\end{array}$ & $0.0^{10}$ & $0.0^{10}$ & $\begin{array}{l}72 . \\
7\end{array}$ & $\begin{array}{l}32 . \\
7\end{array}$ & 90. & $0.0^{10}$ & $0.0^{10}$ \\
\hline & 0.0001 & $0.0^{10}$ & $\begin{array}{l}10 \\
0.0\end{array}$ & $\begin{array}{l}10 \\
0.0\end{array}$ & $\begin{array}{l}10 \\
0.0\end{array}$ & $0.0^{10}$ & 0.0 & $\begin{array}{l}72 . \\
7\end{array}$ & $\begin{array}{l}32 . \\
7\end{array}$ & 90. & $\begin{array}{l}10 \\
0.0\end{array}$ & $\begin{array}{l}10 \\
0.0\end{array}$ \\
\hline & 0.001 & $0.0^{10}$ & $\begin{array}{l}10 \\
0.0\end{array}$ & $\begin{array}{l}10 \\
0.0\end{array}$ & $0.0^{10}$ & $0.0^{10}$ & 0.0 & $8^{61 .}$ & $8^{21 .}$ & $0^{80 .}$ & $0.0^{10}$ & $0.0^{10}$ \\
\hline & 0.01 & $0.0^{10}$ & $\begin{array}{l}10 \\
0.0\end{array}$ & $\begin{array}{l}10 \\
0.0\end{array}$ & $\begin{array}{l}10 \\
0.0\end{array}$ & $0.0^{10}$ & $\begin{array}{l}10 \\
0.0\end{array}$ & $\begin{array}{r}69 . \\
1\end{array}$ & 1.8 & $0^{20 .}$ & $\begin{aligned} & 54 . \\
5 & \end{aligned}$ & $5^{85 .}$ \\
\hline & 0.1 & $\begin{array}{l}10 \\
0.0\end{array}$ & $\begin{array}{l}10 \\
0.0\end{array}$ & $\begin{array}{l}10 \\
0.0\end{array}$ & $\begin{array}{l}10 \\
0.0\end{array}$ & $0.0^{10}$ & $\begin{array}{l}10 \\
0.0\end{array}$ & $\begin{array}{l}72 . \\
7\end{array}$ & 3.6 & 7.3 & $\begin{array}{ll} & 18 . \\
2 & \end{array}$ & $0^{40 .}$ \\
\hline (b) & 0.2 & $\begin{array}{l}10 \\
0.0\end{array}$ & $\begin{array}{l}10 \\
0.0\end{array}$ & $\begin{array}{l}10 \\
0.0\end{array}$ & $\begin{array}{l}10 \\
0.0\end{array}$ & $\begin{array}{l}10 \\
0.0^{10}\end{array}$ & $\begin{array}{l}10 \\
0.0\end{array}$ & $\begin{array}{l}72 . \\
7\end{array}$ & 3.6 & 3.6 & $2^{18 .}$ & $8^{41 .}$ \\
\hline $\begin{array}{c}\text { Non-crack } \\
\text { Images }\end{array}$ & 0.3 & $0^{10}$ & $0.0^{10}$ & $\begin{array}{l}10 \\
0.0\end{array}$ & $\begin{array}{l}10 \\
0.0\end{array}$ & 0.0 & $\begin{array}{l}10 \\
0.0\end{array}$ & $9^{70 .}$ & 3.6 & 0.0 & $2^{18 .}$ & $8^{41 .}$ \\
\hline & 0.4 & $\begin{array}{l}10 \\
0.0\end{array}$ & $\begin{array}{l}10 \\
0.0\end{array}$ & $\begin{array}{l}10 \\
0.0\end{array}$ & $\begin{array}{l}10 \\
0.0\end{array}$ & $\begin{array}{l}10 \\
0.0\end{array}$ & $\begin{array}{l}10 \\
0.0\end{array}$ & $\begin{array}{l}70 . \\
9\end{array}$ & 3.6 & 0.0 & $\begin{array}{ll} & 18 . \\
2 & \end{array}$ & $8^{41 .}$ \\
\hline & 0.5 & $\begin{array}{l}10 \\
0.0\end{array}$ & $\begin{array}{l}10 \\
0.0\end{array}$ & $\begin{array}{l}10 \\
0.0\end{array}$ & $\begin{array}{l}10 \\
0.0\end{array}$ & $\begin{array}{l}10 \\
0.0\end{array}$ & $\begin{array}{l}10 \\
0.0\end{array}$ & $\begin{array}{l}72 . \\
7\end{array}$ & 3.6 & 0.0 & $\begin{array}{ll} & 18 . \\
2 & \end{array}$ & $8^{41 .}$ \\
\hline & 0.6 & $\begin{array}{l}10 \\
0.0\end{array}$ & $\begin{array}{l}10 \\
0.0\end{array}$ & $\begin{array}{l}10 \\
0.0\end{array}$ & $\begin{array}{l}10 \\
0.0\end{array}$ & $\begin{array}{l}10 \\
0.0\end{array}$ & $\begin{array}{l}10 \\
0.0\end{array}$ & $\begin{array}{l}72 . \\
7\end{array}$ & 3.6 & 0.0 & $\begin{array}{ll} & 18 . \\
2 & \end{array}$ & $8^{41 .}$ \\
\hline & 0.7 & $\begin{array}{l}10 \\
0.0\end{array}$ & $\begin{array}{l}10 \\
0.0\end{array}$ & $\begin{array}{l}10 \\
0.0\end{array}$ & $\begin{array}{l}10 \\
0.0\end{array}$ & $\begin{array}{l}10 \\
0.0\end{array}$ & $\begin{array}{l}10 \\
0.0\end{array}$ & $\begin{array}{l}72 . \\
7\end{array}$ & 3.6 & 0.0 & $\begin{array}{ll} & 18 . \\
2 & \end{array}$ & $\begin{array}{l}41 . \\
8\end{array}$ \\
\hline & 0.8 & $\begin{array}{l}10 \\
0.0\end{array}$ & $\begin{array}{l}10 \\
0.0\end{array}$ & $\begin{array}{l}10 \\
0.0\end{array}$ & $\begin{array}{l}10 \\
0.0\end{array}$ & $\begin{array}{l}10 \\
0.0\end{array}$ & $\begin{array}{l}10 \\
0.0\end{array}$ & $\begin{array}{l}72 . \\
7\end{array}$ & 3.6 & 0.0 & $\begin{array}{ll} & 18 . \\
2 & \end{array}$ & $8^{41 .}$ \\
\hline & 0.9 & $\begin{array}{l}10 \\
0.0\end{array}$ & $\begin{array}{l}10 \\
0.0\end{array}$ & $\begin{array}{l}10 \\
0.0\end{array}$ & $\begin{array}{l}10 \\
0.0\end{array}$ & $\begin{array}{l}10 \\
0.0\end{array}$ & $\begin{array}{l}10 \\
0.0\end{array}$ & $\begin{array}{l}72 . \\
7\end{array}$ & 3.6 & 0.0 & $\begin{array}{ll} & 18 . \\
2 & \end{array}$ & $\begin{array}{l}41 . \\
8\end{array}$ \\
\hline & 1.0 & $\begin{array}{l}10 \\
0.0\end{array}$ & $\begin{array}{l}10 \\
0.0\end{array}$ & $\begin{array}{l}10 \\
0.0\end{array}$ & $\begin{array}{l}10 \\
0.0\end{array}$ & $\begin{array}{l}10 \\
0.0\end{array}$ & $\begin{array}{l}10 \\
0.0\end{array}$ & $\begin{array}{l}72 . \\
7\end{array}$ & 3.6 & 0.0 & $\begin{array}{ll} & 18 . \\
2 & \end{array}$ & $\begin{array}{ll} & 41 . \\
8 & \end{array}$ \\
\hline
\end{tabular}


Table 6 Analysis results (\%) by Var. \#1 vs. Var. \#2 (Var. \#3: Gaussian)

\begin{tabular}{|c|c|c|c|c|c|c|c|c|c|c|c|c|}
\hline \multirow{16}{*}{$\begin{array}{c}\text { (a) } \\
\text { Crack } \\
\text { Images }\end{array}$} & $\begin{array}{l}\text { Var. \#2 } \\
\text { Var. \#1 }\end{array}$ & 0.0 & 0.1 & 0.2 & 0.3 & 0.4 & 0.5 & 0.6 & 0.7 & 0.8 & 0.9 & 1.0 \\
\hline & 0 & 0.0 & 0.0 & 0.0 & 0.0 & 9.1 & 34.5 & 76.4 & 90.9 & 96.4 & 98.2 & 96.4 \\
\hline & 0.00001 & 0.0 & 0.0 & 0.0 & 0.0 & 9.1 & 34.5 & 76.4 & 90.9 & 96.4 & 98.2 & 96.4 \\
\hline & 0.0001 & 0.0 & 0.0 & 0.0 & 0.0 & 9.1 & 34.5 & 76.4 & 90.9 & 96.4 & 98.2 & 96.4 \\
\hline & 0.001 & 0.0 & 0.0 & 0.0 & 0.0 & 7.3 & 36.4 & 81.8 & 92.7 & 96.4 & 98.2 & 96.4 \\
\hline & 0.01 & 0.0 & 0.0 & 0.0 & 3.6 & 10.9 & 30.9 & 63.6 & 94.5 & 98.2 & 100.0 & 98.2 \\
\hline & 0.1 & 0.0 & 0.0 & 0.0 & 3.6 & 14.5 & 38.2 & 50.9 & 90.9 & 96.4 & 100.0 & 100.0 \\
\hline & 0.2 & 0.0 & 0.0 & 0.0 & 3.6 & 14.5 & 34.5 & 54.5 & 90.9 & 96.4 & 100.0 & 100.0 \\
\hline & 0.3 & 0.0 & 0.0 & 0.0 & 3.6 & 12.7 & 34.5 & 54.5 & 90.9 & 96.4 & 100.0 & 100.0 \\
\hline & 0.4 & 0.0 & 0.0 & 0.0 & 3.6 & 12.7 & 34.5 & 54.5 & 90.9 & 96.4 & 100.0 & 100.0 \\
\hline & 0.5 & 0.0 & 0.0 & 0.0 & 3.6 & 12.7 & 34.5 & 54.5 & 90.9 & 96.4 & 100.0 & 100.0 \\
\hline & 0.6 & 0.0 & 0.0 & 0.0 & 3.6 & 12.7 & 34.5 & 54.5 & 90.9 & 96.4 & 100.0 & 100.0 \\
\hline & 0.7 & 0.0 & 0.0 & 0.0 & 3.6 & 12.7 & 34.5 & 54.5 & 90.9 & 96.4 & 100.0 & 100.0 \\
\hline & 0.8 & 0.0 & 0.0 & 0.0 & 3.6 & 12.7 & 34.5 & 54.5 & 90.9 & 96.4 & 100.0 & 100.0 \\
\hline & 0.9 & 0.0 & 0.0 & 0.0 & 3.6 & 12.7 & 34.5 & 54.5 & 90.9 & 96.4 & 100.0 & 100.0 \\
\hline & 1.0 & 0.0 & 0.0 & 0.0 & 3.6 & 12.7 & 34.5 & 54.5 & 90.9 & 96.4 & 100.0 & 100.0 \\
\hline \multirow{16}{*}{$\begin{array}{c}\text { (b) } \\
\text { Non-crack } \\
\text { Images }\end{array}$} & Var. \#2 & 0.0 & 0.1 & 0.2 & 0.3 & 0.4 & 0.5 & 0.6 & 0.7 & 0.8 & 0.9 & 1.0 \\
\hline & \multirow{2}{*}{0} & 10 & 10 & 10 & 10 & 10 & 10 & 74. & 45. & 32. & 63. & 94. \\
\hline & & 0.0 & 0.0 & 0.0 & 0.0 & 0.0 & 0.0 & 5 & 5 & 7 & 6 & 5 \\
\hline & \multirow{2}{*}{0.00001} & 10 & 10 & 10 & 10 & 10 & 10 & 74. & 45. & 32. & 63. & 94. \\
\hline & & 0.0 & 0.0 & 0.0 & 0.0 & 0.0 & 0.0 & 5 & 5 & 7 & 6 & 5 \\
\hline & \multirow{2}{*}{0.0001} & 10 & 10 & 10 & 10 & 10 & 10 & 74. & 45. & 32. & 63. & 94. \\
\hline & & 0.0 & 0.0 & 0.0 & 0.0 & 0.0 & 0.0 & 5 & 5 & 7 & 6 & 5 \\
\hline & \multirow{2}{*}{0.001} & 10 & 10 & 10 & 10 & 10 & 10 & 69. & 50. & 30. & 56. & 87. \\
\hline & & 0.0 & 0.0 & 0.0 & 0.0 & 0.0 & 0.0 & 1 & 9 & 9 & 4 & 3 \\
\hline & \multirow{2}{*}{0.01} & 10 & 10 & 98. & 98. & 90. & 85. & 70. & 45. & 23. & 20. & 49. \\
\hline & & 0.0 & 0.0 & 2 & 2 & 9 & 5 & 9 & 5 & 6 & 0 & 1 \\
\hline & \multirow{2}{*}{0.1} & 10 & 10 & 10 & 96. & 94. & 90. & 60. & 21. & \multirow{2}{*}{9.1} & \multirow{2}{*}{9.1} & 29. \\
\hline & & 0.0 & 0.0 & 0.0 & 4 & 5 & 9 & 0 & 8 & & & 1 \\
\hline & \multirow{2}{*}{0.2} & 10 & 10 & 10 & 96. & 96. & 90. & 60. & 20. & \multirow{2}{*}{7.3} & \multirow{2}{*}{9.1} & 29. \\
\hline & & 0.0 & 0.0 & 0.0 & 4 & 4 & 9 & 0 & 0 & & & 1 \\
\hline & 0.3 & 10 & 10 & 10 & 96. & 96. & 90. & 63. & 18. & 3.6 & 7.3 & 25. \\
\hline
\end{tabular}




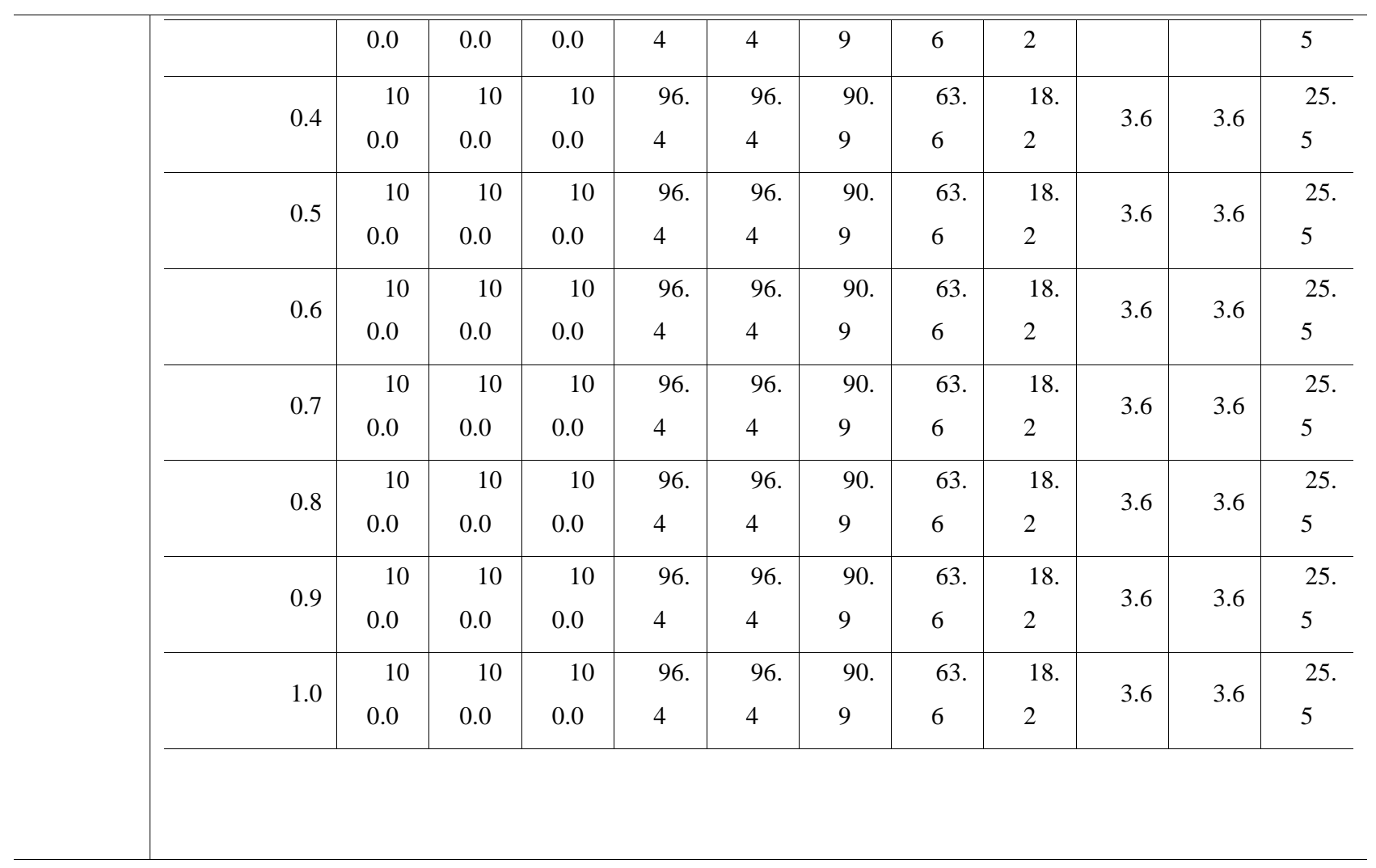


Statistically speaking, Mean was the best in quantitative terms, but its accuracy was somewhat inferior in qualitative terms as shown in Table 7. Table 7 shows the comparison of the results by a statistical method, and the detected cracks are marked with a green outline on the original image. Gaussian statistic is analyzed to be superior in both quantitative and qualitative aspects. The reason for this is analyzed that the neighborhood Gaussian weighted average (Gaussian) rather than the neighborhood local average (Mean) detected the boundary of the crack well.

Table 7 Comparison of results by Var. \#3

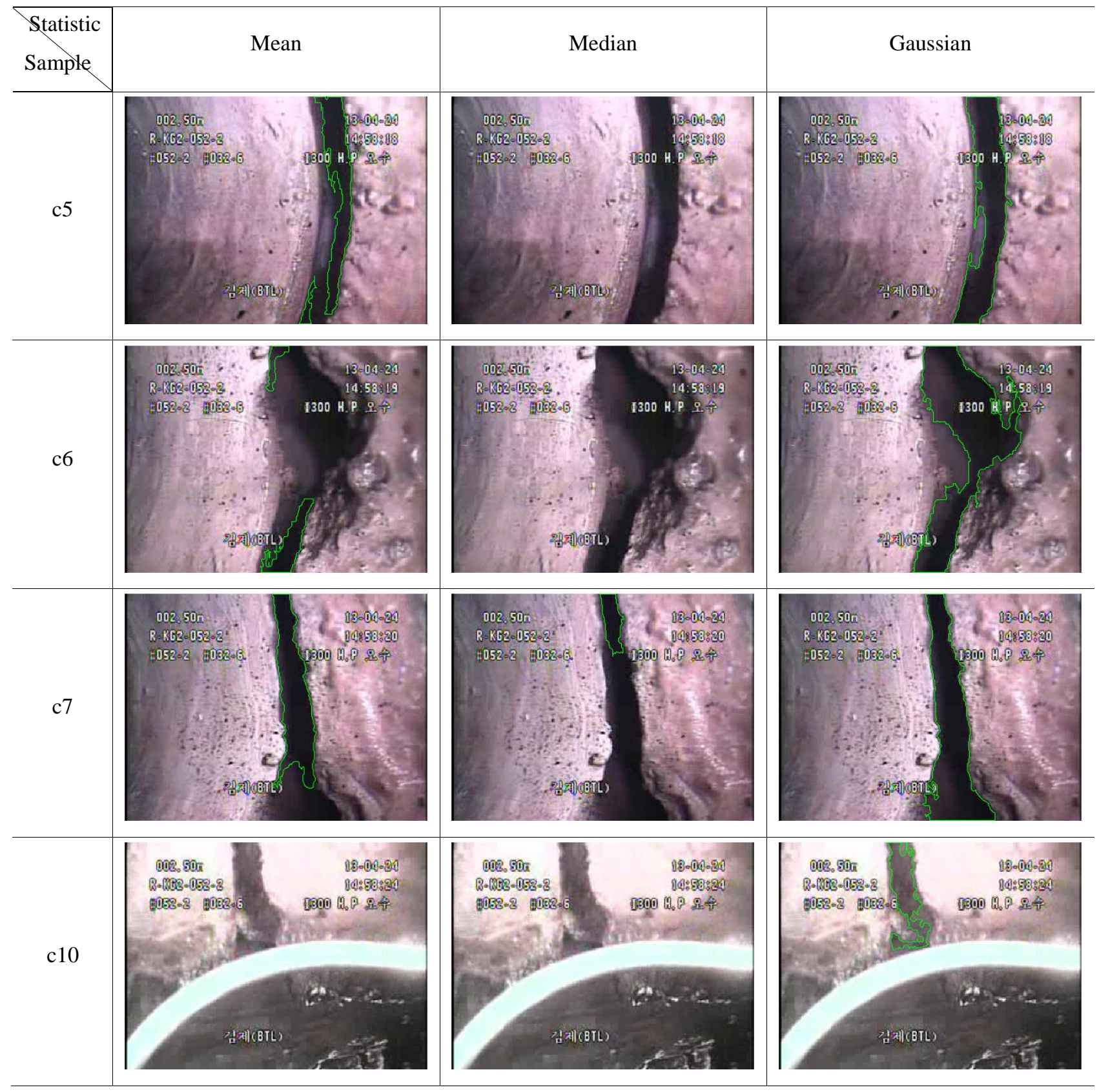




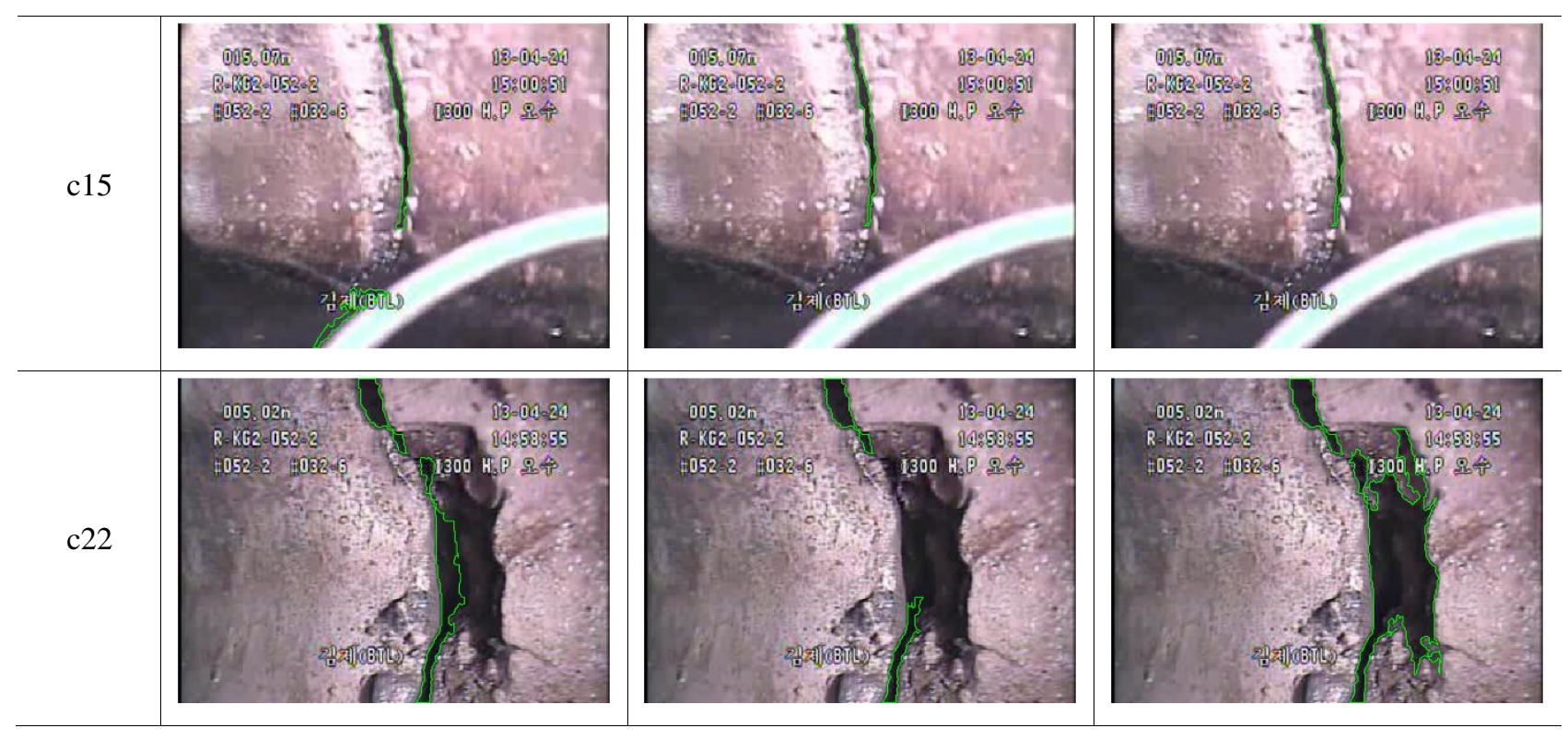




\subsection{User algorithm}

User algorithm is used to find cracks in Step 5 of Chapter 2, and it is a step to find only cracks in Step 4 of Table 2. As shown in Table 8, due to the low-resolution image characteristics of the sewer, it is detected like a crack in black on the left and right (the part marked with 'P'). However, this is due to the nature of the image, and it is not a crack. It should be removed.

Table 8 Application of user algorithm

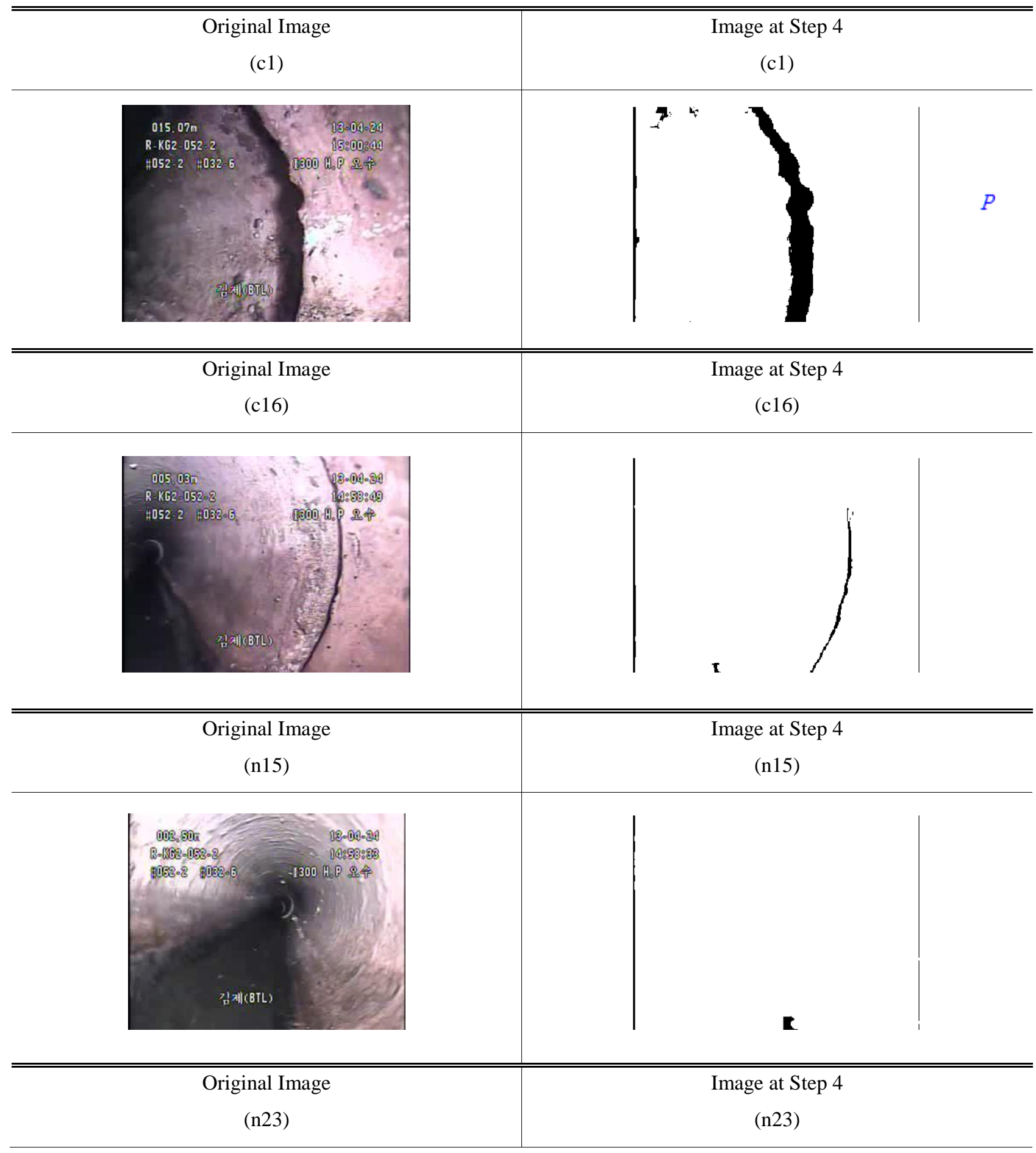




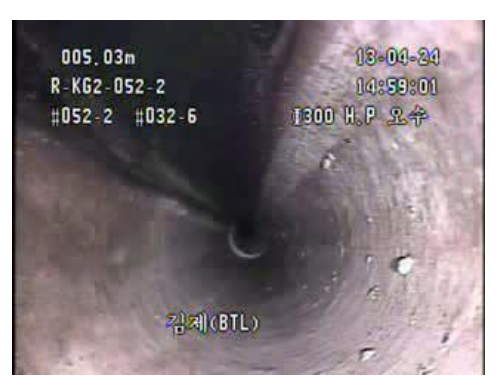

\section{7}

Table 9 User algorithm for crack detection

Input : Image $f(i, j)$ of Step 4

Output : Crack image g(i,j)

$(1 \leq \mathrm{i} \leq \mathrm{sz}(1), 1 \leq \mathrm{j} \leq \mathrm{sz}(2))$

(sz(1) : Height of the image, sz(2) : Width of the image)

\begin{tabular}{|c|c|}
\hline 1 & Use the images $f(j, i)$ of Step 4 \\
\hline 2 & for $(\mathrm{k}=1$ to No. of crack) \\
\hline 3 & $\begin{array}{l}\text { Exclude the most left and the most right rows } \\
\text { (The part maked as ' } \mathrm{P}^{\prime} \text { in Table } 8 \text { )) }\end{array}$ \\
\hline 4 & for $(i=1$ to $s z(1))$ \\
\hline 5 & Compute the crack length \\
\hline 6 & end \\
\hline 7 & for $(j=1$ to $s z(2))$ \\
\hline 8 & Compute crack width \\
\hline 9 & end \\
\hline 10 & $\begin{array}{l}\text { Crack width } \\
\qquad: 3 \mathrm{~mm}(8 \text { pixels }) \text { or greater } \& 160 \text { pixels or } \\
\text { less } \\
\text { Crack length } \\
\quad: 9 \mathrm{~mm}(24 \text { pixels }) \text { or greater }\end{array}$ \\
\hline 11 & end \\
\hline
\end{tabular}


The part marked with 'Q' in Table 8 is barely detected as a crack due to calculation error, but it is not a crack. To remove this error, the crack was detected by judging that the length and the width of the crack should be 9 mm (24 pixels) or more and $3 \mathrm{~mm}$ ( 8 pixels) or more, respectively. Additionally, if the width of the crack was more than half (160 pixels) of 320 pixels, which is the size of the image $(240 \times 320)$, it is determined that it is not a crack. Table 9 shows the algorithm for this step.

\section{Results and analysis}

Through the crack detection procedure presented in Chapter 2 and the optimum parameters and user algorithm presented in Chapter 3, the crack detection results of the low-resolution sewer image were analyzed.

As a result of crack detection from the sewer images, 53 out of 55 (96.4\%) were accurately detected as cracks, and 52 out of $55(94.5 \%)$ were correctly detected as non-cracks. Tables 10 and 11 summarize the results of crack detection from crack and non-crack images, respectively. In Table 10, Bad results refer to detecting non-cracks as cracks, and Good results mean detecting only part of the crack. Excellent results refer to the case of perfect crack detection. Table 11 presents the results of crack detection from non-crack images only. If a crack is detected, when it is a non-crack image, it is considered a bad result. If it is correctly not detected as a crack, then it is an excellent result. Good results are meaningless in this case of non-crack images.

Table 10 Summary of crack images

\begin{tabular}{c|c}
\hline \multirow{2}{*}{ Bad results } & Images \\
& c24, c52 \\
& $(2$ images, 3.6\%) \\
\hline \multirow{2}{*}{ Good results } & $\mathrm{c} 14, \mathrm{c} 16, \mathrm{c} 23, \mathrm{c} 25, \mathrm{c} 31$, \\
& $(10$ images, 19.2\%) \\
\hline \multirow{2}{*}{ Excellent results } & Images other than the above \\
& $(43$ images, 78.2\%) \\
\hline
\end{tabular}

Table 11 Summary of non-crack images

\begin{tabular}{c|c}
\hline & Images \\
\hline Bad results & $\mathrm{n} 3, \mathrm{n} 5, \mathrm{n} 30$ \\
& $(3$ images, 5.4\%) \\
\hline Excellent results & $\begin{array}{c}\text { Images other than the above } \\
\text { (52 images, 94.6\%) }\end{array}$ \\
\hline
\end{tabular}


Table 12 shows the excellent results, and only 12 images are shown in consideration of the paper size. Table 13 shows the good results of detecting only a part of a crack. Table 14 shows the bad results of failing to detect cracks from crack images and false detection of cracks from non-crack images.

Table 12 Excellent results of crack images (Only 12 results)
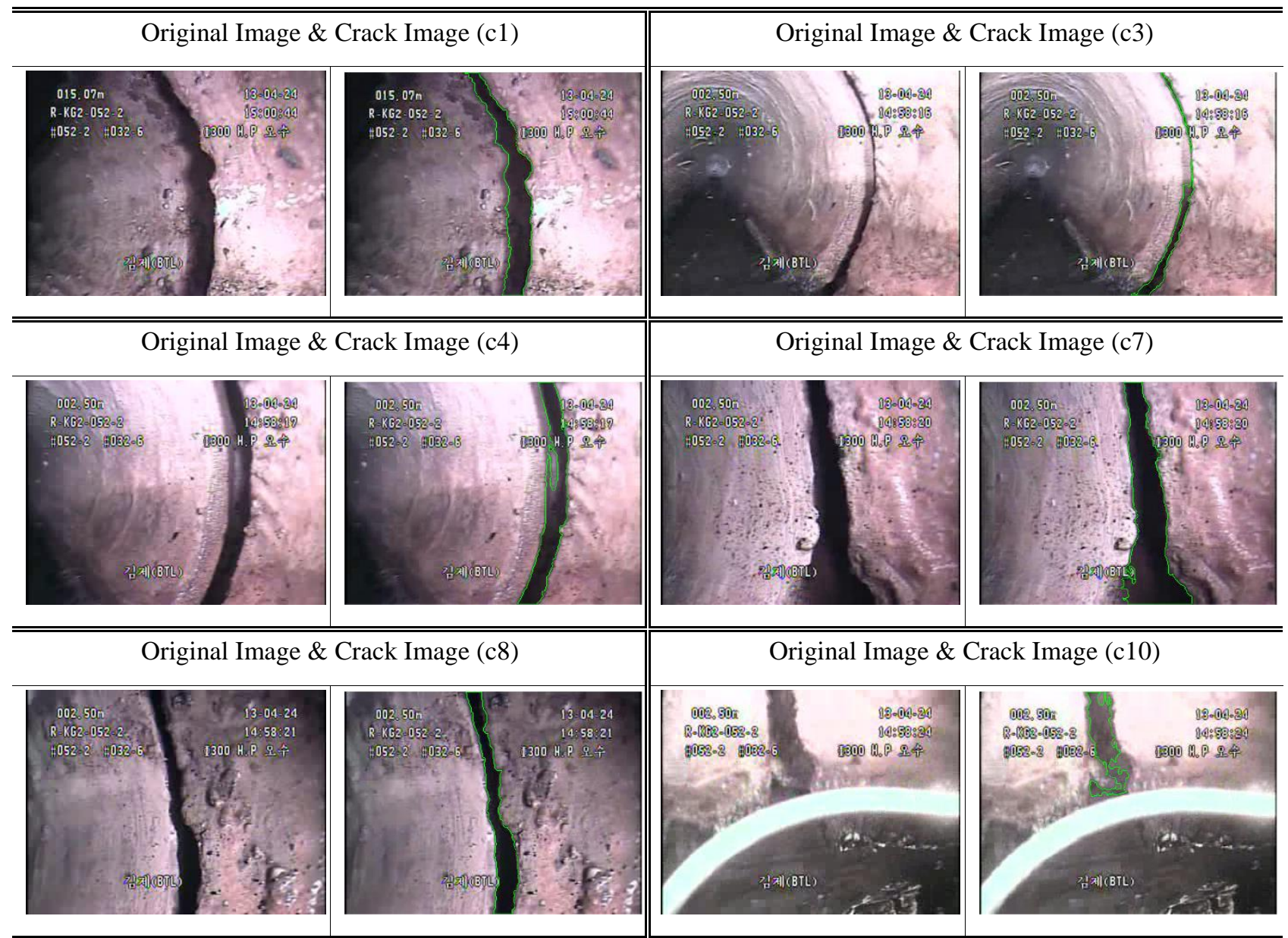

\section{(1)}

Original Image \& Crack Image (c10)

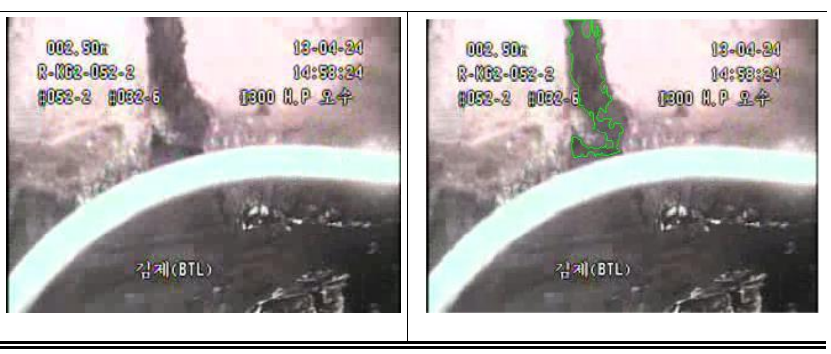

Original Image \& Crack Image (c15)

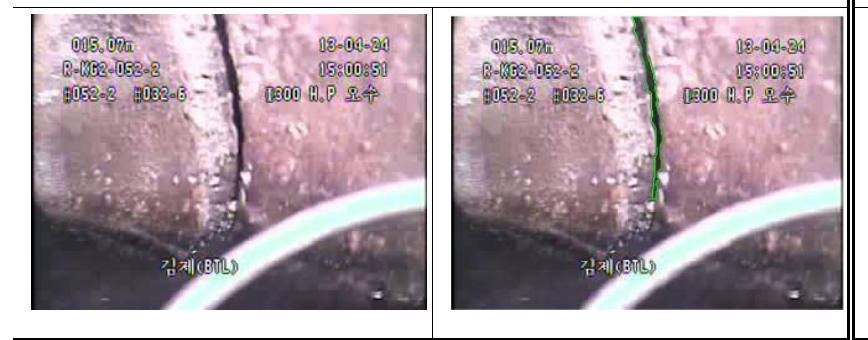

Original Image \& Crack Image (c20)

Original Image \& Crack Image (c17)

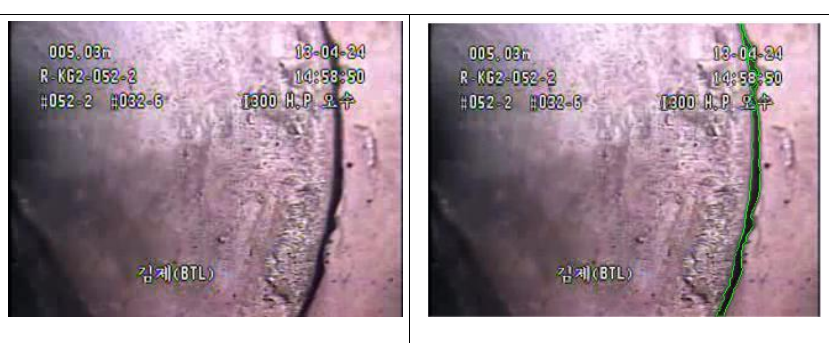

Original Image \& Crack Image (c30) 


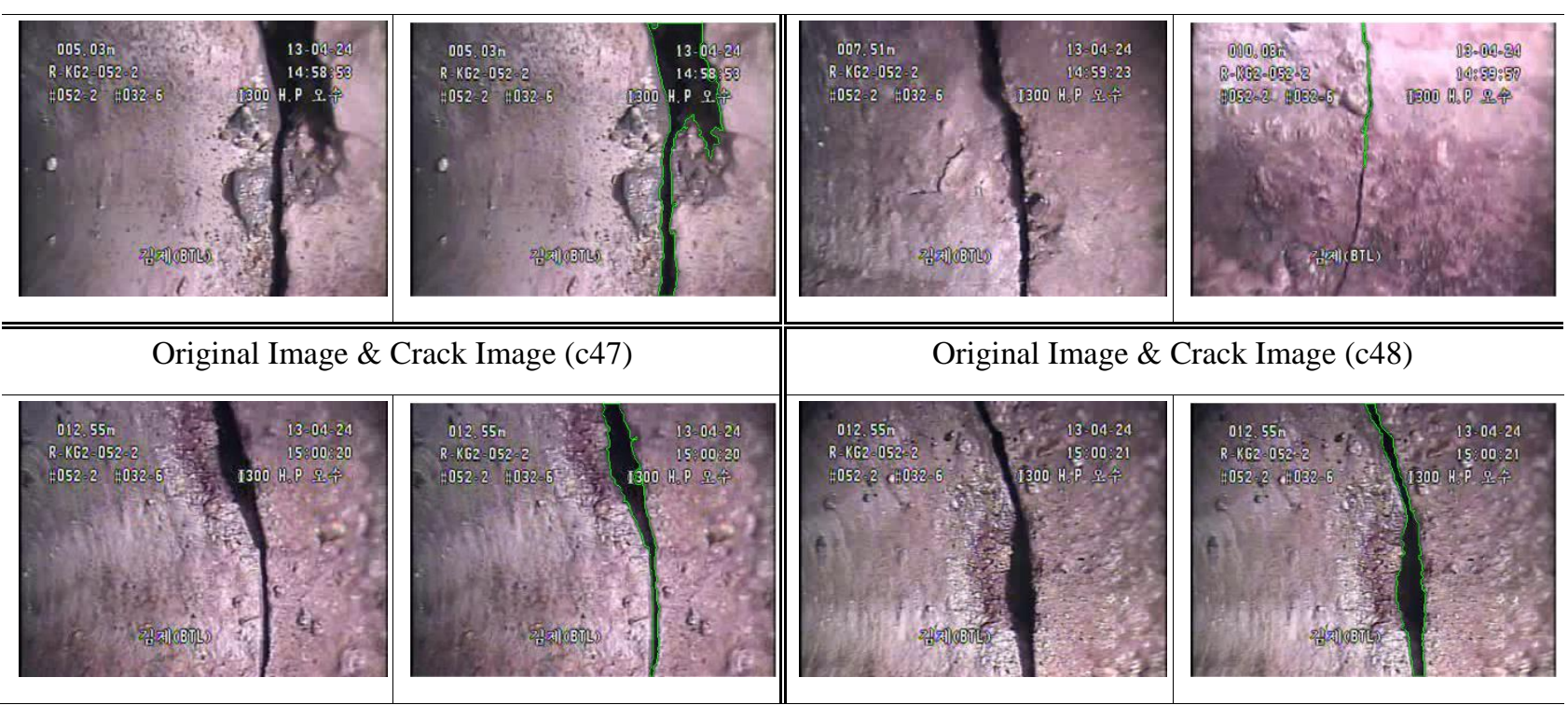


Table 13 Good results of crack images (All results)
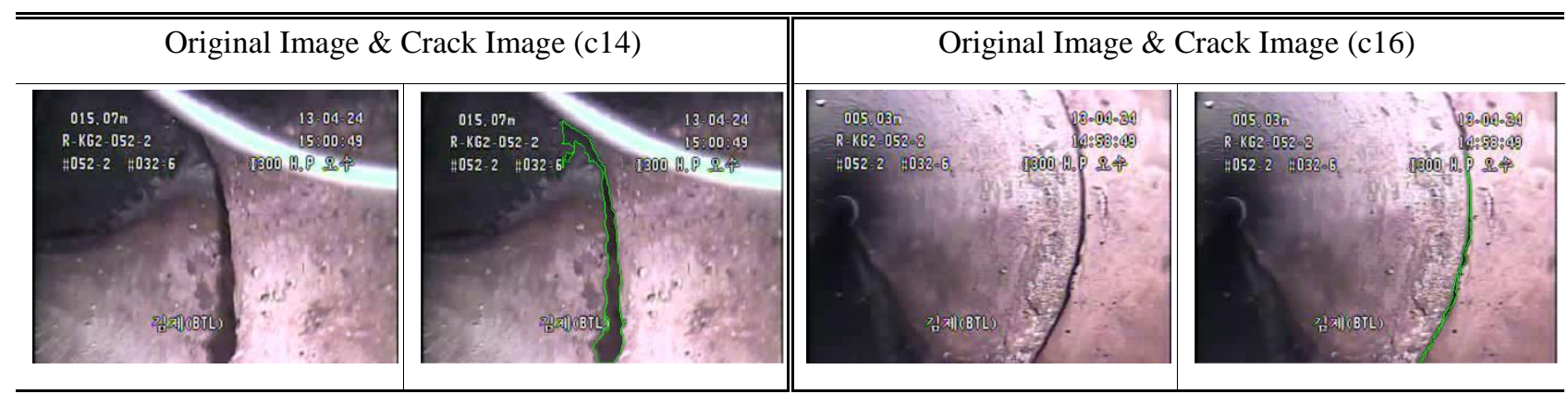

Original Image \& Crack Image (c23)
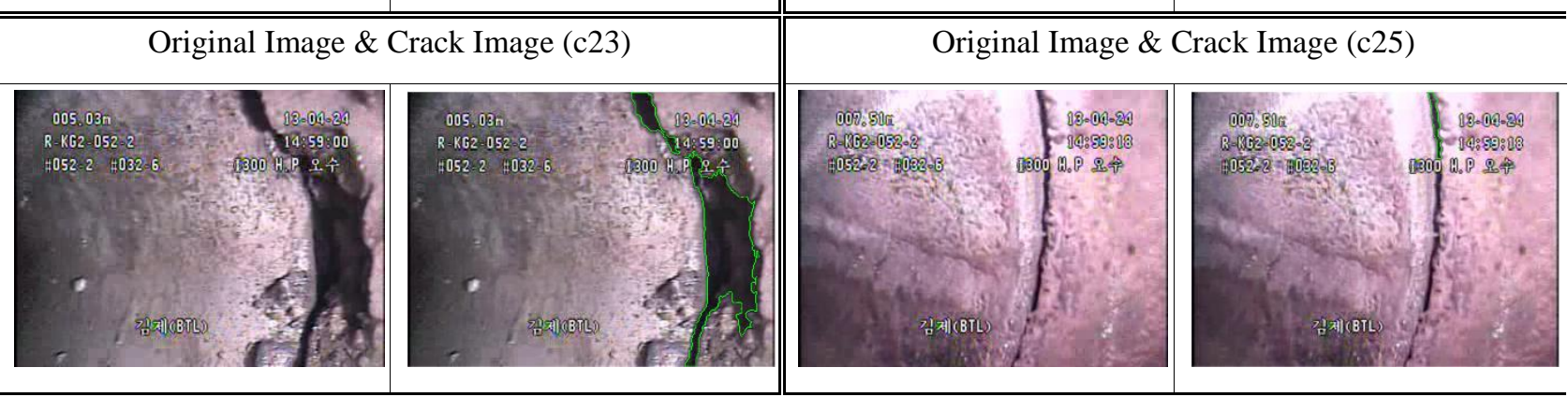

Original Image \& Crack Image (c31)
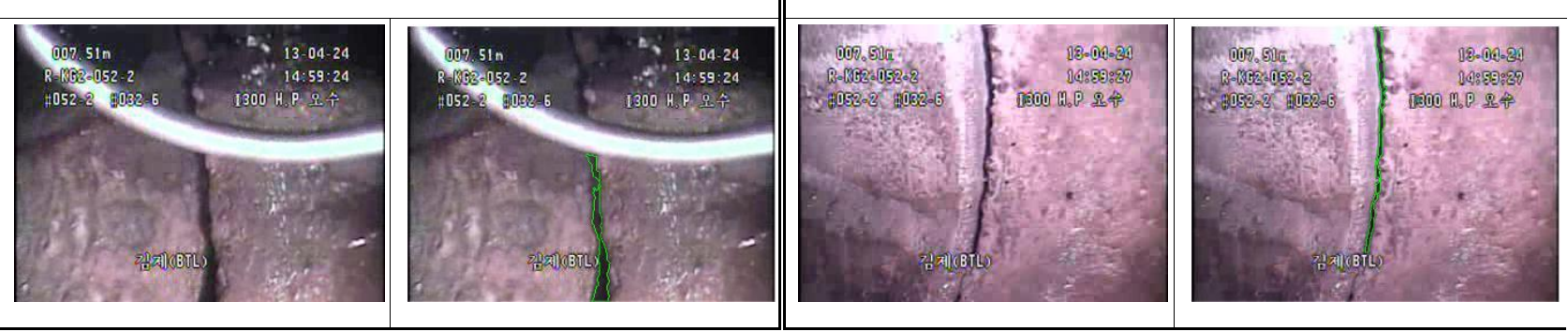

Original Image \& Crack Image (c39)
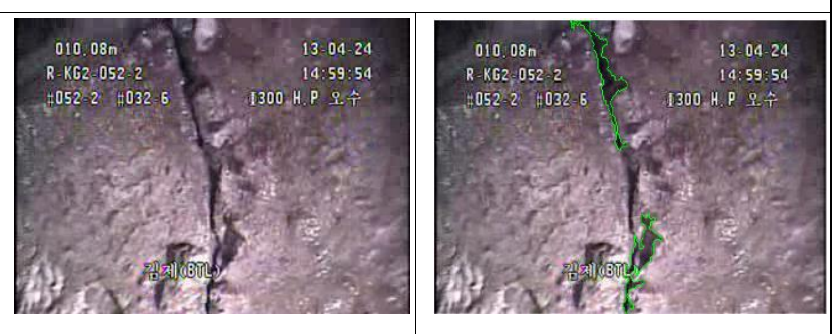

Original Image \& Crack Image (c40)

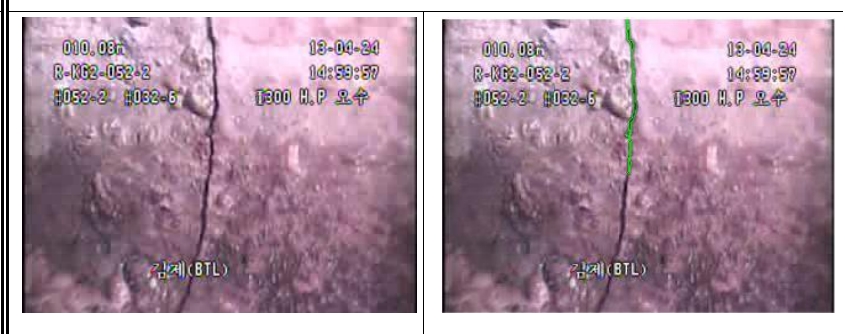

Original Image \& Crack Image (c46)
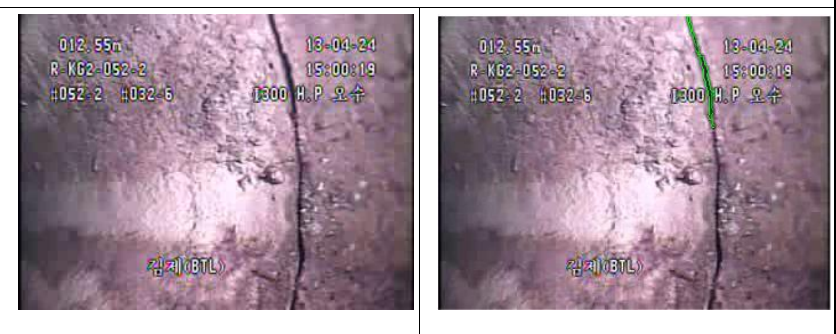

Original Image \& Crack Image (c51)

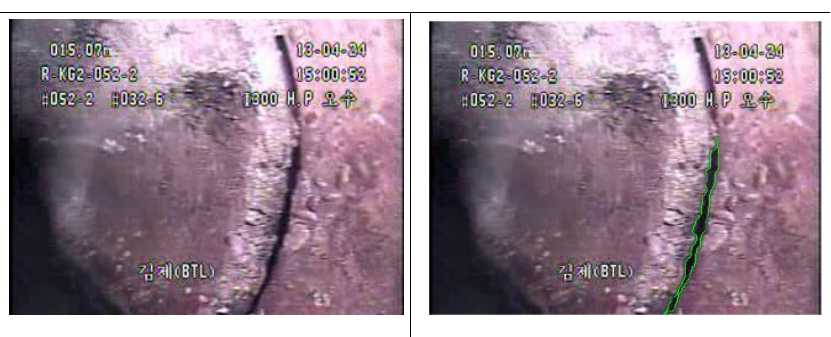


Table 14 Bad results of crack and non-crack images (All results)

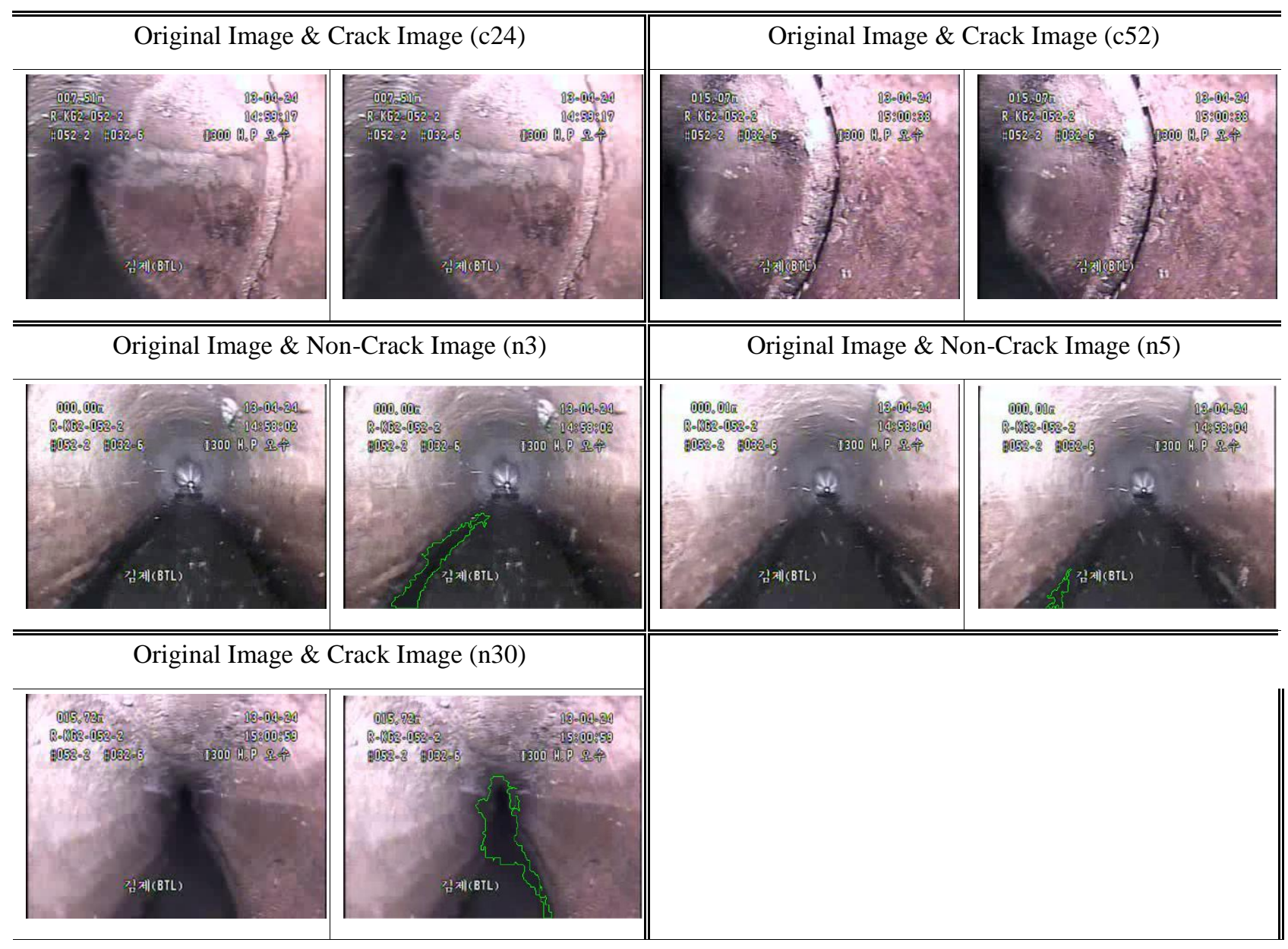

\section{Conclusions}

This study improved the method of automatically detecting cracks from sewer images of very low resolution $(240 \times 320$ $=76,800$ pixels). Cracks were detected through a total of six steps such as enhancing the crack images in Step 2, finding the optimum threshold value in Step 3, and applying an algorithm to detect cracks in Step 5. Cracks were effectively detected by the optimum parameters obtained through Steps 2 and 3 and the user algorithm from Step 5. Despite the low resolution, the detection of crack images and non-crack images showed $96.4 \%$ accuracy and $94.5 \%$ accuracy, respectively. Moreover, the quality was also excellent. The accuracy was much higher than the results of the previous study by Son et al. (2019), and the crack detection procedure was reduced from 8 steps to 6 steps. Therefore, this study significantly advanced the previous research.

$\mathrm{f}$ the resolution of the image is increased (at least 500,000 pixels or more) or an algorithm that effectively removes subtitles from the image is applied, cracks can be detected better. In conclusion, the findings of this study can be effectively used for crack detection of low-resolution images.

\section{ACKNOWLEDGMENT}


This research was supported by the Konyang University Research Fund in 2019.

\section{REFERENCES}

McKim, R. A. and Sinha, S. K. (1999). "Condition assessment of underground sewer pipes using a modified digital image processing paradigm." Tunnelling and Underground Space Technology, Vol. 14, pp. 29-37.

Moselhi, O. and Shehab-Eldeen, T. (1999). "Automated detection of surface defects in water and sewer pipes." Automation in Construction, Vol. 8, No. 5, pp. 581-588.

Sankarasrinivasan, S., Balasubramanian, E., Karthik, K., Chandrasekar, U., and Rishi, G. (2015). "Health monitoring of civil structures with integrated UAV and image processing system." Procedia Computer Science, Vol. 54, pp. 508-515.

Son, B. J., Jeon, J. R., and Heo, G. H. (2017). "Image processing algorithm for crack detection of sewer with low resolution." Journal of the Korea Academia-Industrial Cooperation Society, Vol. 18, No. 2, pp. 590-599.

G. H. Heo, J. R. Jeon and B. J. Son (2019). "Crack Automatic Detection of CCTV Video of Sewer Inspection with Low Resolution." KSCE Journal of Civil Engineering, Vol. 23(3), pp. 1219-1227.

Xu, K., Lxmoore, A. R., and Davies, T. (1998). "Sewer pipe deformation assessment by image analysis of video surveys." Pattern Recognition, Vol. 31, No. 2, pp. 169-180.

Yang, M. D. and Su, T. C. (2008). "Automated diagnosis of sewer pipe defects based on machine learning approaches." Expert Systems with Applications, Vol. 35, No. 3, pp. 1327-1337.

Yang, M. D., Su, T. C., Pan, N. F., and Yang, Y. F. (2011). "Systematic image quality assessment for sewer inspection." Expert Systems with Applications, Vol. 38, No. 3, pp. 1766-1776.

Bradley, D., G. Roth (2007). "Adapting Thresholding Using the Integral Image." Journal of Graphics Tools. Vol. 12, No. 2, pp.13-21.

Zuiderveld, Karel (1994). "Contrast Limited Adaptive Histograph Equalization." Graphic Gems IV. San Diego: Academic Press Professional, 474-48.

Soille, P (1999). "Morphological Image Analysis : Principles and Applications." Springer-Verlag, pp. 173-174.

Gonzalez, R. C., R. E. Woods, and S. L. Eddins (2009). "Digital Image Processing Using MATLAB." Gatesmark Publishing. 